\title{
Dynamic Complexities in 2-Dimensional Discrete-Time Predator-Prey Systems with Allee Effect in the Prey
}

\author{
Jie Yan, ${ }^{1,2}$ Chunli Li, ${ }^{1}$ Xueli Chen, ${ }^{1,2}$ and Lishun Ren ${ }^{1}$ \\ ${ }^{1}$ Department of Mathematics and Statistics, Zhoukou Normal University, Zhoukou, Henan 466000, China \\ ${ }^{2}$ Department of Mathematics and Statistics, Zhengzhou University, Zhengzhou, Henan 450001, China \\ Correspondence should be addressed to Lishun Ren; 9236rls@sina.com
}

Received 6 June 2016; Accepted 18 July 2016

Academic Editor: Massimiliano Ferrara

Copyright (c) 2016 Jie Yan et al. This is an open access article distributed under the Creative Commons Attribution License, which permits unrestricted use, distribution, and reproduction in any medium, provided the original work is properly cited.

\begin{abstract}
The Allee effect is incorporated into a predator-prey model with linear functional response. Compared with the predator-prey which only takes the crowding effect and predator partially dependent on prey into consideration, it is found that the Allee effect of the prey species would increase the extinction risk of both the prey and predator. Moreover, by using a center manifold theorem and bifurcation theory, it is shown that the model with Allee effect undergoes the flip bifurcation and Hopf bifurcation in the interior of $R_{+}^{2}$ with different Allee effect values. In the two bifurcations, we can come to the conclusion that different Allee effect will have different bifurcation value and the increasing of the Allee effect will increase the value of bifurcation, respectively.
\end{abstract}

\section{Introduction}

The predator-prey interaction is a topic of great interest for many ecologists and mathematics. Such interactions have motivated various models of phenotypic coevolution in preypredator communities. Many researchers have studied the dynamical behavior of the predator-prey system in ecology and contributed to growth of continuous models for large size population [1-13]. Also, a number of researchers have established the discrete-time models which are more appropriate and provided more efficient results as compared to the continuous models for small size population.

In [14], the author has investigated the stability and bifurcation of discrete-time predator-prey system with crowding effect of predator where predator is partially dependent on prey, this can be described by the following system (3). By analyzing, the author got the following conclusion: the systems undergoes flip bifurcation and Hopf bifurcation at fixed points under specific conditions when $\delta$ varies in small neighborhood. And numerical simulations of the model display cascade of period-doubling bifurcation in orbits of periods 2, 4, 8, chaotic sets and stable window of period 9 orbit in case of flip bifurcation, and smooth invariant circles in case of Hopf bifurcation. In biology, it means that the predator coexists with prey at period- $n$ orbits and smooth invariant circle.

Now, on the basis of previous systems, we make an improvement in it. We assume the prey with Allee effect and use center manifold theorem and bifurcation theory to study the system with Allee effect.

The concept of Allee effect originates from observations made by an American ecologist Allee in the 1930s [15]. In the 1930s, Allee found that factors affecting the growth rate of single group include the resources space constraints and density of the initial population density. When the initial density of the population is lower than a certain critical value, population will eventually become extinct because of the restriction of the conditions or other conditions of the population reproduction, which is called Allee effect. This effect is probably one of the most exciting concepts in ecology, a concept that has long been neglected but now has attracted renewed interest. The most usual continuous growth function considering Allee effect is expressed by [16-19]

$$
\frac{d x}{d t}=r x\left(1-\frac{x}{k}\right)(x-m),
$$

where $x$ denotes the population density; $k$ denotes the carrying capacity; $r$ denotes the intrinsic per capita growth 
rate of the population. In (1), when $m>0$, it is the strong Allee effect. when $-k<m \leq 0$, it is the weak Allee effect. Weak Allee effect growth function always remains positive, while in the strong Allee effect, there is a threshold population level $m$ below which the growth is negative and the species will become extinct.

Other mathematical forms have been proposed to model the Allee effect [16, 20-23] and most of them are topologically equivalent [24]. For instance, in [25, 26] the following equation is proposed:

$$
\frac{d x}{d t}=r\left(1-\frac{x}{k}-\frac{n}{x+b}\right)
$$

which has been called additive Allee effect; (2) allows the modelling of multiple Allee effect [20]. Here, $n$ and $b$ are Allee effect parameter.

In our paper, we consider the following system:

$$
\begin{aligned}
& \dot{x}(t)=a x\left(1-\frac{x}{k}\right)-\frac{b x y}{x+l}, \\
& \dot{y}(t)=c y+\frac{m b x y}{x+l}-d y^{2},
\end{aligned}
$$

where all the parameters are positive. $x(t)$ and $y(t)$ represent the densities of prey and predator population, respectively; $a, b$ denote the intrinsic growth rate of prey and predator, respectively; $c$ denotes the growth rate of predator due to alternative resources. Again, $d$ denotes competition among individuals of predator species due to overcrowding; $l$ denotes the half saturation constant; $m$ denotes the conversion rate for predator; and $k$ denotes carrying capacity of the prey in a particular habitat.

In this prey-predator system, due to the impact of their own breeding process or because of the prey itself, the prey population will have the Allee effect. By observing some signs, single species of plant and animal which are influenced by Allee effect are very wide. Many facts point out the fact that the prey of the growth rate with Allee effect will fall to very low but remain positive. So, Allee effect provides an important basis for the management of endangered species. Without the Allee effect, the evolution of the system is a sublimate situation, the time to reach the $p$ point of the system is less than that of the system without Allee system which is not conducive to the evolution of biological, under the influence of the Allee effect, and the evolution of the system will reach a state of equilibrium. In our paper, it is shown that Allee effect of the prey species has a significant effect on the dynamics of the predator-prey system with linear functional response. Many studies have demonstrated the potential importance of Allee effect for dynamics of small population, range expansion, community composition, and biological invasion. It is widely accepted that Allee effect may increase the extinction risk of low density populations.

Because of the difficulties in finding mates when the prey population density becomes low, the Allee effect may occur in the prey species. So, in this paper, we consider the following Allee effect [27]. For example, this might correspond to the density below which it is so difficult to find a mate that reproduction does not compensate for mortality. Let $f(x)$ be the fertility of a species that has $x$ adults in an isolated patch; then the fertility increases with the population density, which is described by

$$
f(x)=\frac{a x}{A_{1}+x}
$$

where $a$ is the maximum per capita birth rate of the species, $x /\left(A_{1}+x\right)$ is the per capita fertilization probability of the species, and $A_{1}$ is the Allee effect constant of the species. Here, if $A_{1}>0$, it is strong Allee effect; the fertility of the species will be therefore zero when $x$ is zero and approaches to $a$ when $x$ becomes very large. Then, $f(x)$ increases with $x$, which depends on the parameter $A_{1}$. Biologically, $A_{1}$ is the population density at which a species reaches half its maximum fertility. If $A_{1}<0$, it is the weak Allee effect. When $A_{1}=0$, the fertility is density independent with $f(x)=a$.

Now, we apply forward Euler's scheme to the system of (3); we choose a time step size $\delta>0$. For any $t \geq 0$, let $x_{t}=x(t)$, $y_{t}=y(t), x_{t+1}=x(t+\delta)$, and $y_{t+1}=y(t+\delta)$, since

$$
\begin{aligned}
& \dot{x}=\lim _{\delta \rightarrow 0} \frac{x_{t+1}-x_{t}}{\delta}, \\
& \dot{y}=\lim _{\delta \rightarrow 0} \frac{y_{t+1}-y_{t}}{\delta} .
\end{aligned}
$$

We can assume that for small enough $\delta>0$

$$
\begin{aligned}
& \dot{x}=\frac{x_{t+1}-x_{t}}{\delta}, \\
& \dot{y}=\frac{y_{t+1}-y_{t}}{\delta} .
\end{aligned}
$$

Thus, from system (1), we get the discrete-time system as

$$
\begin{aligned}
& x \longrightarrow x+\delta\left[a x\left(1-\frac{x}{k}\right)-\frac{b x y}{x+l}\right], \\
& y \longrightarrow y+\delta\left(c y+\frac{m b x y}{x+l}-d y^{2}\right),
\end{aligned}
$$

where $\delta$ is the step size. Shrinking the step size in Euler's method will yield numerical solutions that more accurately approximate the true solution.

So, when the prey species is subject to Allee effect

$$
f(x)=\frac{a x}{A_{1}+x},
$$

the system of (7) can transform the following system:

$$
\begin{aligned}
& x \longrightarrow x+\delta x\left[\left(1-\frac{x}{k}\right) \frac{a x}{A_{1}+x}-\frac{b y}{x+l}\right], \\
& y \longrightarrow y+\delta y\left(c+\frac{m b x}{x+l}-d y\right),
\end{aligned}
$$

where $A_{1}$ is Allee effect threshold value, if $A_{1}>0$, the Allee effect will be strong, and if $A_{1}<0$, the Allee effect will be weak. Here $A_{1}$ is a positive parameter denoting Allee 
effect imposed on the prey population. Next, we investigate model (9) by way of mathematical analysis and numerical simulations.

Keeping in view of the literature, the rest of the paper is organized as follows: Section 2 deals with the stability criterion of the system at different fixed points in interior of $R_{+}^{2}$. In Section 3, we obtain the specific conditions of existence of flip bifurcation and Hopf bifurcation. Numerical simulations are presented to support our analytical findings in Section 4, especially for the period doubling bifurcation and chaotic behavior. Finally a brief conclusion is given in the last section.

\section{Stability of Fixed Points}

The fixed points of system (9) are $p_{0}(0,0), p_{1}(k, 0), p_{2}(0, c / d)$, and $p_{3}\left(x^{*}, y^{*}\right)$, where $x^{*}, y^{*}$ satisfy

$$
\begin{aligned}
a\left(1-\frac{x^{*}}{k}\right) \frac{x^{*}}{A_{1}+x^{*}}-\frac{b y^{*}}{x^{*}+l} & =0, \\
c+\frac{m b x^{*}}{x^{*}+l}-d y^{*} & =0 .
\end{aligned}
$$

The Jacobian matrix of $(9)$ at the fixed point $(x, y)$ is written as

$$
J=\left(\begin{array}{cc}
1+\delta\left[\frac{a x^{2}+2 A_{1} a x}{\left(A_{1}+x\right)^{2}}-\frac{2 a x^{3}+3 A_{1} a x^{2}}{k\left(A_{1}+x\right)^{2}}-\frac{b l y}{(x+l)^{2}}\right. & -\frac{\delta b x}{x+l} \\
\frac{\delta m b l y}{(x+l)^{2}} & 1+\delta\left(c+\frac{m b x}{x+l}-2 d y\right)
\end{array}\right)
$$
by

The characteristic equation of the Jacobian matrix is given

$$
\lambda^{2}+p(x, y) \lambda+q(x, y)=0
$$

where

$$
\begin{aligned}
& p(x, y)=-\operatorname{tr} J=-2-\delta\left[\frac{a x^{2}+2 A_{1} a x}{\left(A_{1}+x\right)^{2}}\right. \\
& \left.-\frac{2 a x^{3}+3 A_{1} a x^{2}}{k\left(A_{1}+x\right)^{2}}-\frac{b l y}{(x+l)^{2}}+c+\frac{m b x}{x+l}-2 d y\right] \\
& q(x, y)=\operatorname{det} J=1+\delta\left[\frac{a x^{2}+2 A_{1} a x}{\left(A_{1}+x\right)^{2}}\right. \\
& \left.-\frac{2 a x^{3}+3 A_{1} a x^{2}}{k\left(A_{1}+x\right)^{2}}-\frac{b l y}{(x+l)^{2}}\right][1 \\
& \left.+\delta\left(c+\frac{m b x}{x+l}-2 d y\right)\right]+\frac{\delta^{2} m b^{2} l x y}{(x+l)^{3}} .
\end{aligned}
$$

Clearly, we can see the Jacobian matrix of (9) at the point $p_{0}(0,0)$ is

$$
J=\left(\begin{array}{cc}
1 & 0 \\
0 & 1+\delta c
\end{array}\right) .
$$

So, $\lambda_{1}=1, \lambda_{2}=1+\delta c$; then $p_{0}(0,0)$ is unstable.

Now, we state a definition and a lemma same as in $[28,29]$.

Definition 1. A fixed point $(x, y)$ is called

(i) sink if $\left|\lambda_{1}\right|<1$ and $\left|\lambda_{2}\right|<1$, and it is asymptotically stable;

(ii) source if $\left|\lambda_{1}\right|>1$ and $\left|\lambda_{2}\right|>1$, and it is locally unstable; (iii) saddle if $\left|\lambda_{1}\right|>1$ and $\left|\lambda_{2}\right|<1$ (or $\left|\lambda_{1}\right|<1$ and $\left|\lambda_{2}\right|>$ $1)$;

(iv) nonhyperbolic if either $\left|\lambda_{1}\right|=1$ or $\left|\lambda_{2}\right|=1$.

Lemma 2. Let $F(\lambda)=\lambda^{2}+P \lambda+Q$. Suppose that $F(1)>0$ and $\lambda_{1}, \lambda_{2}$ are roots of $F(\lambda)=0$. Then, one has the following:

(i) $\left|\lambda_{1}\right|<1$ and $\left|\lambda_{2}\right|<1$ if and only if $F(-1)>0$ and $Q<1$

(ii) $\left|\lambda_{1}\right|<1$ and $\left|\lambda_{2}\right|>1$ (or $\left|\lambda_{1}\right|>1$ and $\left|\lambda_{2}\right|<1$ ) if and only if $F(-1)<0$;

(iii) $\left|\lambda_{1}\right|>1$ and $\left|\lambda_{2}\right|>1$ if and only if $F(-1)>0$ and $Q>1$;

(iv) $\lambda_{1}=-1$ and $\left|\lambda_{2}\right| \neq 1$ if and only if $F(-1)=0$ and $P \neq 0,2$;

(v) $\left|\lambda_{1}\right|$ and $\left|\lambda_{2}\right|$ are complex and $\left|\lambda_{1}\right|=-1,\left|\lambda_{2}\right|=-1$ if and only if $P^{2}-4 Q<0$ and $Q=1$.

Proposition 3. The fixed point $p_{1}(k, 0)$ is source if $\delta>2 / a+$ $2 A_{1} / a k$, saddle if $0<\delta<2 / a+2 A_{1} / a k$, and nonhyperbolic if $\delta=2 / a+2 A_{1} / a k$. In this condition, we assume $A_{1}>-k$. That is, if $-k<A_{1}<0$, it is weak Allee effect, and if $A_{1}>0$, it is strong Allee effect.

One can see that when $\delta=2 / a+2 A_{1} / a k$, one of the eigen values of fixed point $p_{1}(k, 0)$ is -1 and magnitude of other is not equal to 1 . Thus the flip bifurcation occurs when a parameter changes in small neighborhood of $\delta=2 / a+$ $2 A_{1} / a k$.

Proposition 4. There exist different topological types of $p_{2}(0, c / d)$ for possible parameters.

(i) $p_{2}(0, c / d)$ is $\operatorname{sink}$ if $0<\delta<\min \{2 / c, 2 d l / b c\}$.

(ii) $p_{2}(0, c / d)$ is source if $\delta>\max \{2 / c, 2 d l / b c\}$.

(iii) $p_{2}(0, c / d)$ is nonhyperbolic if $\delta=2 / c$ or $\delta=2 d l / b c$. 
(iv) $p_{2}(0, c / d)$ is saddle for all value of the parameters, except for values which lie in (i) to (iii).

In this condition, we can see that the system at $p_{2}$ is not affected by the Allee effect threshold value; that is, bifurcation parameter $\delta$ has nothing to do with $A_{1}$.

The term (iii) of Proposition 4 implies that the parameters lie in the set

$$
\begin{aligned}
F_{A} & =\left\{\left(a, b, c, d, k, l, m, \delta, A_{1}\right), \delta=\frac{2}{c}, \delta\right. \\
& \left.\neq \frac{2 d l}{b c}, a, b, c, d, k, l, m, \delta, A_{1}>0\right\} .
\end{aligned}
$$

If the term (iii) of Proposition 4 holds, then one of the eigen values of fixed points $p_{2}(0, c / d)$ is -1 and the magnitude of the other is not equal to 1 . The point $p_{2}(0, c / d)$ undergoes flip bifurcation when the parameter changes in small neighborhood of $F_{A}$.

Last condition, the characteristic equation of the Jacobian matrix J of system (9) at the fixed point $p_{3}\left(x^{*}, y^{*}\right)$ is written as

$$
\lambda^{2}+p\left(x^{*}, y^{*}\right) \lambda+q\left(x^{*}, y^{*}\right)=0
$$

where

$$
\begin{aligned}
& p\left(x^{*}, y^{*}\right)=-2-G \delta, \\
& q\left(x^{*}, y^{*}\right)=1+G \delta+H \delta^{2}, \\
& G=\frac{a x^{* 2}+2 A_{1} a x^{*}}{\left(A_{1}+x^{*}\right)^{2}}-\frac{2 a x^{* 3}+3 A_{1} a x^{* 2}}{k\left(A_{1}+x^{*}\right)^{2}} \\
& \quad-\frac{b l y^{*}}{\left(x^{*}+l\right)^{2}}+c+\frac{m b x^{*}}{x^{*}+l}-2 d y^{*}, \\
& H=\left[\frac{a x^{* 2}+2 A_{1} a x^{*}}{\left(A_{1}+x^{*}\right)^{2}}-\frac{2 a x^{* 3}+3 A_{1} a x^{* 2}}{k\left(A_{1}+x^{*}\right)^{2}}\right. \\
& \left.\quad-\frac{b l y^{*}}{\left(x^{*}+l\right)^{2}}\right]\left[c+\frac{m b x^{*}}{x^{*}+l}-2 d y^{*}\right]+\frac{m b^{2} l x^{*} y^{*}}{\left(x^{*}+l\right)^{3}} .
\end{aligned}
$$

Now

$$
F(\lambda)=\lambda^{2}-(2+G \delta) \lambda+\left(1+G \delta+H \delta^{2}\right) .
$$

Therefore

$$
\begin{gathered}
F(1)=H \delta^{2}, \\
F(-1)=4+2 G \delta+H \delta^{2} .
\end{gathered}
$$

Using Lemma 2, we get the following proposition.

Proposition 5. There exist different topological types of $p_{3}\left(x^{*}, y^{*}\right)$ for all possible parameters.

(i) $p_{3}\left(x^{*}, y^{*}\right)$ is a sink if either condition (1) or (2) holds:

(1) $G^{2}-4 H \geq 0$ and $0<\delta<\left(-G-\sqrt{G^{2}-4 H}\right) / H$,

(2) $G^{2}-4 H<0$ and $0<\delta<-G / H$. (ii) $p_{3}\left(x^{*}, y^{*}\right)$ is a source if either condition (1) or (2) holds:

(1) $G^{2}-4 H \geq 0$ and $\delta>\left(-G+\sqrt{G^{2}-4 H}\right) / H$,

(2) $G^{2}-4 H<0$ and $\delta>-G / H$.

(iii) $p_{3}\left(x^{*}, y^{*}\right)$ is nonhyperbolic if either condition (1) or (2) holds:

(1) $G^{2}-4 H \geq 0$ and $\delta=\left(-G \pm \sqrt{G^{2}-4 H}\right) / H$,

(2) $G^{2}-4 H<0$ and $\delta=-G / H$.

(iv) $p_{3}\left(x^{*}, y^{*}\right)$ is saddle for all values of the parameters, except for values which lie in (i) to (iii).

If the term (1) in (iii) of Proposition 5 holds, then one of the eigen values of the fixed point $p_{3}\left(x^{*}, y^{*}\right)$ is -1 and the magnitude of the other is not equal to 1 . The term (1) in (iii) of Proposition 5 may be written as follows:

$$
\begin{aligned}
F_{B 1} & =\left\{\left(a, b, c, d, k, l, m, \delta, A_{1}\right): \delta\right. \\
& =\frac{-G-\sqrt{G^{2}-4 H}}{H}, G^{2}-4 H \\
& \left.\geq 0, a, b, c, d, k, l, m, \delta, A_{1}>0\right\}, \\
F_{B 2} & =\left\{\left(a, b, c, d, k, l, m, \delta, A_{1}\right): \delta\right. \\
& =\frac{-G+\sqrt{G^{2}-4 H}}{H}, G^{2}-4 H \\
\geq & \left.0, a, b, c, d, k, l, m, \delta, A_{1}>0\right\} .
\end{aligned}
$$

Then a flip bifurcation will occur in the neighborhood of $F_{B 1}$ or $F_{B 2}$.

If the term (2) in (iii) of Proposition 5 holds, then the eigen values of the fixed point $p_{3}\left(x^{*}, y^{*}\right)$ are a pair of complex conjugate numbers with modulus 1 . The term (2) in (iii) of Proposition 5 may be written as follows:

$$
\begin{aligned}
H_{B} & =\left\{\left(a, b, c, d, k, l, m, \delta, A_{1}\right): \delta=-\frac{G}{H}, G^{2}-4 H\right. \\
& \left.<0, a, b, c, d, k, l, m, \delta, A_{1}>0\right\} .
\end{aligned}
$$

One can see that a Hopf bifurcation will occur in the neighborhood of $H_{B}$.

\section{Bifurcation Behavior}

In this section, we study the flip bifurcation and Hopf bifurcation at the fixed point $p_{3}\left(x^{*}, y^{*}\right)$. 
3.1. Flip Bifurcation. We consider system (9) with arbitrary parameter $\left(a, b, c, d, k, l, m, \delta_{1}, A_{1}\right) \in F_{B 1}$, which is described as follows:

$$
\begin{aligned}
& x \longrightarrow x+\delta_{1} x\left[\left(1-\frac{x}{k}\right) \frac{a x}{A_{1}+x}-\frac{b y}{x+l}\right], \\
& y \longrightarrow y+\delta_{1} y\left(c+\frac{m b x}{x+l}-d y\right),
\end{aligned}
$$

$p_{3}\left(x^{*}, y^{*}\right)$ is fixed point of system (22), where $x^{*}, y^{*}$ are given by (9), and

$$
\delta_{1}=\frac{-G-\sqrt{G^{2}-4 H}}{H} .
$$

The eigen values of $p_{3}\left(x^{*}, y^{*}\right)$ are $\lambda_{1}=-1, \lambda_{2}=3+G \delta_{1}$, with $\left|\lambda_{2}\right| \neq 1$ by Proposition 5 .

Consider the perturbation of (22) as below:

$$
\begin{aligned}
& x \longrightarrow x+\left(\delta_{1}+\delta^{*}\right) x\left[\left(1-\frac{x}{k}\right) \frac{a x}{A_{1}+x}-\frac{b y}{x+l}\right], \\
& y \longrightarrow y+\left(\delta_{1}+\delta^{*}\right) y\left(c+\frac{m b x}{x+l}-d y\right),
\end{aligned}
$$

where $\left|\delta^{*}\right| \ll 1$ is a limited perturbation parameter.

Let $u=x-x^{*}$ and $v=y-y^{*}$.

After the transformation of the fixed point $p_{3}\left(x^{*}, y^{*}\right)$ of system (24) to the point $(0,0)$, we obtain

$$
\begin{aligned}
& \left(\begin{array}{l}
u \\
v
\end{array}\right) \\
& \longrightarrow\left(\begin{array}{c}
a_{11} u+a_{12} v+a_{13} u^{2}+a_{14} u v+b_{11} \delta^{*} u+b_{12} \delta^{*} v+b_{13} \delta^{*} u^{2}+b_{14} \delta^{*} u v+O\left(|u|,|v|,\left|\delta^{*}\right|\right)^{3} \\
a_{21} u+a_{22} v+a_{23} u^{2}+a_{24} u v+a_{25} v^{2}+b_{21} \delta^{*} u+b_{22} \delta^{*} v+b_{23} \delta^{*} u^{2}+b_{24} \delta^{*} u v+b_{25} \delta^{*} v^{2}+O\left(|u|,|v|,\left|\delta^{*}\right|\right)^{3}
\end{array}\right),
\end{aligned}
$$

where

$$
\begin{aligned}
& a_{11}=1+\delta_{1}\left[\frac{\left(2 A_{1}+x^{*}\right) a x^{*}}{\left(A_{1}+x^{*}\right)^{2}}-\frac{\left(3 A_{1} x^{* 2}+2 x^{* 3}\right) a}{k\left(A_{1}+x^{*}\right)^{2}}\right. \\
& \left.-\frac{b y^{*}}{x^{*}+l}+\frac{b x^{*} y^{*}}{\left(x^{*}+l\right)^{2}}\right] \\
& a_{12}=-\frac{\delta_{1} b x^{*}}{x^{*}+l}, \\
& a_{13}=\delta_{1}\left[\frac{a A_{1}^{2}}{\left(A_{1}+x^{*}\right)^{3}}-\frac{\left(a x^{* 3}+3 A_{1} x^{*}+3 A_{1}^{2} x^{*}\right)}{k\left(A_{1}+x^{*}\right)^{3}}\right. \\
& \left.+\frac{b y^{*}}{\left(x^{*}+l\right)^{2}}-\frac{b x^{*} y^{*}}{\left(x^{*}+l\right)^{3}}\right] \\
& a_{14}=\delta_{1}\left[\frac{b x^{*}}{\left(x^{*}+l\right)^{2}}-\frac{b}{x^{*}+l}\right] \text {, } \\
& b_{11}=\frac{\left(2 A_{1}+x^{*}\right) a x^{*}}{\left(A_{1}+x^{*}\right)^{2}}-\frac{\left(3 A_{1} x^{* 2}+2 x^{* 3}\right) a}{k\left(A_{1}+x^{*}\right)^{2}} \\
& -\frac{b y^{*}}{x^{*}+l}+\frac{b x^{*} y^{*}}{\left(x^{*}+l\right)^{2}} \text {, } \\
& b_{12}=-\frac{b x^{*}}{x^{*}+l}, \\
& b_{13}=\frac{a A_{1}^{2}}{\left(A_{1}+x^{*}\right)^{3}}-\frac{a x^{* 3}+3 A_{1} x^{*}+3 A_{1}^{2} x^{*}}{k\left(A_{1}+x^{*}\right)^{3}}
\end{aligned}
$$

$$
\begin{aligned}
& +\frac{b y^{*}}{\left(x^{*}+l\right)^{2}}-\frac{b x^{*} y^{*}}{\left(x^{*}+l\right)^{3}}, \\
b_{14} & =\frac{b x^{*}}{\left(x^{*}+l\right)^{2}}-\frac{b}{x^{*}+l}, \\
a_{21} & =\frac{\delta_{1} m b l y^{*}}{\left(x^{*}+l\right)^{2}}, \\
a_{22} & =1-\delta_{1} d y^{*}, \\
a_{23} & =-\frac{\delta_{1} m b l y^{*}}{\left(x^{*}+l\right)^{3}}, \\
a_{24} & =\frac{\delta_{1} m b l}{\left(x^{*}+l\right)^{2}}, \\
a_{25} & =-\delta_{1} d, \\
b_{21} & =\frac{m b l y^{*}}{\left(x^{*}+l\right)^{2}}, \\
b_{22} & =-d y^{*}, \\
b_{25} & =-d \\
b_{23} & =-\frac{m b l y^{*}}{\left(x^{*}+l\right)^{3}}, \\
& =\frac{m b l}{\left(x^{*}+l\right)^{2}}, \\
b^{2} &
\end{aligned}
$$

Consider the following translation:

$$
\left(\begin{array}{l}
u \\
v
\end{array}\right)=T\left(\begin{array}{l}
\tilde{x} \\
\tilde{y}
\end{array}\right),
$$


where

$$
\begin{aligned}
T & =\left(\begin{array}{cc}
a_{11} & a_{12} \\
-1-a_{11} & \lambda_{2}-a_{11}
\end{array}\right), \\
T^{-1} & =\left(\begin{array}{cc}
\frac{\lambda_{2}-a_{11}}{a_{12}\left(\lambda_{2}+1\right)} & -\frac{1}{\lambda_{2}+1} \\
\frac{1+a_{11}}{a_{12}\left(\lambda_{2}+1\right)} & \frac{1}{\lambda_{2}+1}
\end{array}\right), \\
\left(\begin{array}{l}
u \\
v
\end{array}\right) & =T\left(\begin{array}{l}
\tilde{x} \\
\tilde{y}
\end{array}\right)=\left(\begin{array}{cc}
a_{11} & a_{12} \\
-1-a_{11} & \lambda_{2}-a_{11}
\end{array}\right)\left(\begin{array}{l}
\tilde{x} \\
\tilde{y}
\end{array}\right) .
\end{aligned}
$$

Taking $T^{-1}$ on both sides of (16), we get

$$
\left(\begin{array}{l}
\tilde{x} \\
\tilde{y}
\end{array}\right) \longrightarrow\left(\begin{array}{cc}
-1 & 0 \\
0 & \lambda_{2}
\end{array}\right)\left(\begin{array}{l}
\tilde{x} \\
\tilde{y}
\end{array}\right)+\left(\begin{array}{l}
f\left(u, v, \delta^{*}\right) \\
g\left(u, v, \delta^{*}\right)
\end{array}\right),
$$

where

$$
\begin{aligned}
f\left(u, v, \delta^{*}\right)= & \frac{\left[a_{13}\left(\lambda_{2}-a_{11}\right)-a_{12} a_{23}\right] u^{2}}{a_{12}\left(\lambda_{2}+1\right)} \\
& +\frac{\left[a_{14}\left(\lambda_{2}-a_{11}\right)-a_{12} a_{24}\right] u v}{a_{12}\left(\lambda_{2}+1\right)} \\
& -\frac{a_{12} a_{25} v^{2}}{a_{12}\left(\lambda_{2}+1\right)} \\
& +\frac{\left[b_{11}\left(\lambda_{2}-a_{11}\right)-a_{12} b_{21}\right] \delta^{*} u}{a_{12}\left(\lambda_{2}+1\right)} \\
& +\frac{\left[b_{12}\left(\lambda_{2}-a_{11}\right)-a_{12} b_{22}\right] \delta^{*} v}{a_{12}\left(\lambda_{2}+1\right)} \\
& +\frac{\left[b_{13}\left(\lambda_{2}-a_{11}\right)-a_{12} b_{23}\right] \delta^{*} u^{2}}{a_{12}\left(\lambda_{2}+1\right)} \\
& +\frac{\left[b_{14}\left(\lambda_{2}-a_{11}\right)-a_{12} b_{24}\right] \delta^{*} u v}{a_{12}\left(\lambda_{2}+1\right)} \\
& -\frac{a_{12} b_{25} \delta^{*} v^{2}}{a_{12}\left(\lambda_{2}+1\right)}+0\left(|u|,|v|,\left|\delta^{*}\right|\right)^{3} \\
&
\end{aligned}
$$

Apply the center manifold theorem to (22) at the origin in the limited neighborhood of $\delta^{*}=0$. The center manifold $W^{c}(0,0)$ can be approximately presented as

$$
\begin{aligned}
& W^{c}(0,0)=\left\{(\tilde{x}, \tilde{y}): \tilde{y}=a_{0} \delta^{*}+a_{1} \tilde{x}^{2}+a_{2} \tilde{x} \delta^{*}\right. \\
& \left.+a_{3} \delta^{* 2}+O\left(\left(|\tilde{x}|+\left|\delta^{*}\right|\right)^{3}\right)\right\},
\end{aligned}
$$

where $O\left(|\widetilde{x}|+\left|\delta^{*}\right|\right)^{3}$ is a function with at least third orders in variables $\left(\tilde{x}, \delta^{*}\right)$.

By simple calculations for center manifold, we have

$$
\begin{aligned}
& a_{0}=0, \\
& a_{1}=\frac{\left[a_{13}\left(1+a_{11}\right)+a_{12} a_{23}\right] a_{12}-\left[a_{14}\left(1+a_{11}\right)+a_{12} a_{24}\right]\left(1+a_{11}\right)+a_{25}\left(1+a_{11}\right)^{2}}{1-\lambda_{2}^{2}}, \\
& a_{2}=-\frac{\left[b_{11}\left(1+a_{11}\right)+a_{12} b_{21}\right] a_{12}+\left[b_{12}\left(1+a_{11}\right)+a_{12} b_{22}\right]\left(1+a_{11}\right)}{a_{12}\left(1+\lambda_{2}\right)^{2}}, \\
& a_{3}=0 .
\end{aligned}
$$


Now, consider the map restricted to the center manifold $W^{c}(0,0)$ as below:

$$
\begin{aligned}
f: \tilde{x} \longrightarrow & -\tilde{x}+h_{1} \tilde{x}^{2}+h_{2} \tilde{x} \delta^{*}+h_{3} \tilde{x}^{2} \delta^{*}+h_{4} \tilde{x}\left(\delta^{*}\right)^{2} \\
& +h_{5} \tilde{x}^{3}+O\left(\left(|\tilde{x}|+\left|\delta^{*}\right|\right)^{4}\right),
\end{aligned}
$$

where

$$
\begin{aligned}
& h_{1}=\frac{\left[a_{13}\left(\lambda_{2}-a_{11}\right)-a_{12} a_{23}\right] a_{12}}{\lambda_{2}+1} \\
& -\frac{\left[a_{14}\left(\lambda_{2}-a_{11}\right)-a_{12} a_{24}\right]\left(1+a_{11}\right)}{\lambda_{2}+1} \\
& -\frac{a_{12} a_{25}\left(1+a_{11}\right)^{2}}{a_{12}\left(\lambda_{2}+1\right)} \\
& h_{2}=\frac{\left[b_{11}\left(\lambda_{2}-a_{11}\right)-a_{12} b_{21}\right]}{\lambda_{2}+1} \\
& -\frac{\left[b_{12}\left(\lambda_{2}-a_{11}\right)-a_{12} b_{22}\right]\left(1+a_{11}\right)}{\left(\lambda_{2}+1\right) a_{12}}, \\
& h_{3}=\frac{\left[a_{13}\left(\lambda_{2}-a_{11}\right)-a_{12} a_{23}\right] 2 a_{2} a_{12}}{\lambda_{2}+1} \\
& +\frac{\left[a_{14}\left(\lambda_{2}-a_{11}\right)-a_{12} a_{24}\right]\left(\lambda_{2}-2 a_{11}-1\right) a_{2}}{\lambda_{2}+1} \\
& +\frac{2 a_{25}\left(1+a_{11}\right)\left(\lambda_{2}-a_{11}\right) a_{2}}{\lambda_{2}+1} \\
& +\frac{\left[b_{11}\left(\lambda_{2}-a_{11}\right)-a_{12} b_{21}\right] a_{1}}{\lambda_{2}+1} \\
& +\frac{\left[b_{12}\left(\lambda_{2}-a_{11}\right)-a_{12} b_{22}\right]\left(\lambda_{2}-a_{11}\right) a_{1}}{a_{12}\left(\lambda_{2}+1\right)} \\
& +\frac{\left[b_{13}\left(\lambda_{2}-a_{11}\right)-a_{12} b_{23}\right] a_{12}}{\lambda_{2}+1} \\
& -\frac{\left[b_{14}\left(\lambda_{2}-a_{11}\right)-a_{12} b_{24}\right]\left(1+a_{11}\right)}{\lambda_{2}+1} \\
& -\frac{b_{25}\left(1+a_{11}\right)^{2}}{\lambda_{2}+1} \\
& h_{4}=\frac{\left[b_{11}\left(\lambda_{2}-a_{11}\right)-a_{12} b_{21}\right] a_{2}}{\lambda_{2}+1} \\
& +\frac{\left[b_{12}\left(\lambda_{2}-a_{11}\right)-a_{12} b_{22}\right]\left(\lambda_{2}-a_{11}\right) a_{2}}{a_{12}\left(\lambda_{2}+1\right)}, \\
& h_{5}=\frac{\left[a_{13}\left(\lambda_{2}-a_{11}\right)-a_{12} a_{23}\right] 2 a_{12} a_{1}}{\lambda_{2}+1} \\
& +\frac{\left[a_{14}\left(\lambda_{2}-a_{11}\right)-a_{12} a_{24}\right]\left(\lambda_{2}-2 a_{11}-1\right) a_{1}}{\lambda_{2}+1} \\
& +\frac{2 a_{25}\left(1+a_{11}\right)\left(\lambda_{2}-a_{11}\right) a_{1}}{\lambda_{2}+1}
\end{aligned}
$$

According to flip bifurcation, the discriminatory quantities $\gamma_{1}$ and $\gamma_{2}$ are given by

$$
\begin{aligned}
& \gamma_{1}=\left.\left(\frac{\partial^{2} f}{\partial \tilde{x} \partial \delta^{*}}+\frac{1}{2} \frac{\partial f}{\partial \delta^{*}} \frac{\partial^{2} f}{\partial \tilde{x}^{2}}\right)\right|_{(0,0)}, \\
& \gamma_{2}=\left.\left(\frac{1}{6} \frac{\partial^{3} f}{\partial \tilde{x}^{3}}+\left(\frac{1}{2} \frac{\partial^{2} f}{\partial \tilde{x}^{2}}\right)^{2}\right)\right|_{(0,0)} .
\end{aligned}
$$

After simple calculations, we obtain $\gamma_{1}=h_{2}$ and $\gamma_{2}=$ $h_{5}+h_{1}^{2}$.

Analyzing above and the flip bifurcation conditions discussed in [30], we write the following theorem.

Theorem 6. If $\gamma_{2} \neq 0$ and parameter $\delta^{*}$ alters in the limiting region of the point $(0,0)$, then system (10) passes through flip bifurcation at the point $p_{3}\left(x^{*}, y^{*}\right)$. Also, the period-2 points that bifurcations from fixed point $p_{3}\left(x^{*}, y^{*}\right)$ are stable (resp., unstable) if $\gamma_{2}>0$ (resp., $\left.\gamma_{2}<0\right)$.

3.2. Hopf Bifurcation. Consider system (7) with arbitrary parameter $\left(a, b, c, k, l, m, \delta_{2}, A_{1}\right) \in H_{B}$. This is described as follows:

$$
\begin{aligned}
& x \longrightarrow x+\delta_{2} x\left[\left(1-\frac{x}{k}\right) \frac{a x}{A_{1}+x}-\frac{b y}{x+l}\right], \\
& y \longrightarrow y+\delta_{2} y\left(c+\frac{m b x}{x+l}-d y\right) .
\end{aligned}
$$

$p_{3}\left(x^{*}, y^{*}\right)$ is the fixed point of system (37), where $x^{*}, y^{*}$ are given by (3) and $\delta_{2}=-G / H$.

Consider the perturbation of (37) as given below:

$$
\begin{aligned}
& x \longrightarrow x+\left(\delta_{2}+\delta\right) x\left[\left(1-\frac{x}{k}\right) \frac{a x}{A_{1}+x}-\frac{b y}{x+l}\right], \\
& y \longrightarrow y+\left(\delta_{2}+\delta\right) y\left(c+\frac{m b x}{x+l}-d y\right),
\end{aligned}
$$

where $|\delta| \ll 1$ is limited perturbation parameter.

The characteristic equation of map (38) at $p_{3}\left(x^{*}, y^{*}\right)$ is given by $\lambda^{2}+p(\delta) \lambda+q(\delta)=0$, where

$$
\begin{aligned}
& p(\delta)=-2-G \delta, \\
& q(\delta)=1+G \delta+H \delta^{2} .
\end{aligned}
$$

Since the parameter $\left(a, b, c, k, l, m, \delta_{2}, A_{1}\right) \in H_{B}$, the eigen values of $p_{3}\left(x^{*}, y^{*}\right)$ are a pair of complex conjugate numbers $\bar{\lambda}$ and $\lambda$ with modulus 1 by Proposition 5 , where

$$
\bar{\lambda}, \lambda=\frac{-p(\delta) \mp i \sqrt{4 q(\delta)-p^{2}(\delta)}}{2} .
$$

Therefore

$$
\bar{\lambda}, \lambda=1+\frac{G\left(\delta_{2}+\delta\right)}{2} \mp \frac{i\left(\delta_{2}+\delta\right) \sqrt{4 H-G^{2}}}{2} .
$$


Now we have

$$
\begin{aligned}
& |\lambda|=q(\delta)^{1 / 2}, \\
& l=\left.\frac{d|\lambda|}{d \delta}\right|_{\delta=0}=-\frac{G}{2}>0 .
\end{aligned}
$$

When $\delta$ varies in limited neighborhood of $\delta=0$, then $\bar{\lambda}, \lambda=$ $\alpha \pm i \beta$, where

$$
\begin{aligned}
& \alpha=1+\frac{\delta_{2} G}{2}, \\
& \beta=\frac{\delta_{2} \sqrt{4 H-G^{2}}}{2} .
\end{aligned}
$$

Hopf bifurcation requires that when $\delta=0$, then $\overline{\lambda^{n}}, \lambda^{n} \neq$ $1(n=1,2,3,4)$ which is equivalent to $p(0) \neq-2,0,1,2$.

Since the parameter $\left(a, b, c, d, l, m, \delta_{2}, A_{1}\right) \in H_{B}$. therefore $p(0) \neq-2,2$. It is the only requirement that $p(0) \neq 0,1$, which follows that $G^{2} \neq 2 H, 3 H$. Let $u=x-x^{*}$ and $v=y-y^{*}$.

After the transformation of the fixed points $p_{3}\left(x^{*}, y^{*}\right)$ of system (38) to the points $(0,0)$, we have

$$
\begin{aligned}
& \left(\begin{array}{l}
u \\
v
\end{array}\right) \\
& \longrightarrow\left(\begin{array}{c}
a_{11} u+a_{12} v+a_{13} u^{2}+a_{14} u v+0(|u|,|v|)^{3} \\
a_{21} u+a_{22} v+a_{23} u^{2}+a_{24} u v+a_{25} v^{2}+0(|u|,|v|)^{3}
\end{array}\right),
\end{aligned}
$$

where $a_{11}, a_{12}, a_{13}, a_{21}, a_{22}, a_{23}, a_{24}, a_{25}$ are given in (26) by substituting $\delta_{2}$ for $\delta_{2}+\delta$.

Now, we discuss the normal form of (44) when $\delta=0$.

Consider the translation as below:

$$
\left(\begin{array}{l}
u \\
v
\end{array}\right)=T\left(\begin{array}{l}
\tilde{x} \\
\tilde{y}
\end{array}\right)
$$

where

$$
\begin{aligned}
T & =\left(\begin{array}{cc}
a_{12} & 0 \\
\alpha-a_{11} & -\beta
\end{array}\right), \\
T^{-1} & =\left(\begin{array}{cc}
\frac{1}{a_{12}} & 0 \\
\frac{\alpha-a_{11}}{\beta a_{12}} & -\frac{1}{\beta}
\end{array}\right), \\
\left(\begin{array}{l}
u \\
v
\end{array}\right) & =T\left(\begin{array}{l}
\tilde{x} \\
\tilde{y}
\end{array}\right)=\left(\begin{array}{cc}
a_{12} & 0 \\
\alpha-a_{11} & -\beta
\end{array}\right)\left(\begin{array}{l}
\tilde{x} \\
\tilde{y}
\end{array}\right) .
\end{aligned}
$$

Taking $T^{-1}$ on both sides of (44), we get

$$
\left(\begin{array}{l}
\tilde{x} \\
\tilde{y}
\end{array}\right) \longrightarrow\left(\begin{array}{cc}
\alpha & -\beta \\
\beta & \alpha
\end{array}\right)\left(\begin{array}{l}
\tilde{x} \\
\tilde{y}
\end{array}\right)+\left(\begin{array}{l}
\tilde{f}(\tilde{x}, \tilde{y}) \\
\tilde{g}(\tilde{x}, \tilde{y})
\end{array}\right),
$$

where

$$
\begin{aligned}
\tilde{f}(\tilde{x}, \tilde{y})= & \frac{a_{13}}{a_{12}} u^{2}+\frac{a_{14}}{a_{12} u v}+O(|u|,|v|)^{3}, \\
\widetilde{g}(\tilde{x}, \tilde{y})= & \frac{\left[a_{13}\left(\alpha-a_{11}\right)-a_{12} a_{23}\right]}{a_{12} \beta} u^{2} \\
& +\frac{\left[a_{14}\left(\alpha-a_{11}\right)-a_{12} a_{24}\right]}{a_{12} \beta} u v-\frac{a_{25}}{\beta} v^{2} \\
& +O(|u|,|v|)^{3} \\
u= & a_{12} \tilde{x}, \\
v= & \left(\alpha-a_{11}\right) \tilde{x}-\beta \widetilde{y} .
\end{aligned}
$$

Now

$$
\begin{aligned}
& \tilde{f}_{\tilde{x} \tilde{x}}=2 a_{12} a_{13}+2 a_{14}\left(\alpha-a_{11}\right), \\
& \tilde{f}_{\tilde{x} \tilde{y}}=-a_{14} \beta \text {, } \\
& \tilde{f}_{\tilde{y} \tilde{y}}=0 \text {, } \\
& \tilde{f}_{\tilde{x} \tilde{x} \tilde{x}}=0 \text {, } \\
& \widetilde{f}_{\tilde{x} \tilde{x} \tilde{y}}=0 \text {, } \\
& \widetilde{f}_{\tilde{x} \tilde{y} \tilde{y}}=0 \text {, } \\
& \tilde{f}_{\tilde{y} \tilde{y} \tilde{y}}=0 \text {, } \\
& \tilde{g}_{\tilde{x} \tilde{x}}=\frac{2 a_{12}}{\beta}\left[a_{13}\left(\alpha-a_{11}\right)-a_{12} a_{23}\right] \\
& +\frac{2\left(\alpha-a_{11}\right)}{\beta}\left[a_{14}\left(\alpha-a_{11}\right)-a_{12} a_{24}\right] \\
& -\frac{2 a_{25}}{\beta}\left(\alpha-a_{11}\right)^{2} \text {, } \\
& \tilde{g}_{\tilde{x} \tilde{y}}=-\left[a_{14}\left(\alpha-a_{11}\right)-a_{12} a_{24}\right]+2 a_{25}\left(\alpha-a_{11}\right) \text {, } \\
& \tilde{g}_{\tilde{y} \tilde{y}}=-2 a_{25} \beta \text {, } \\
& \tilde{g}_{\tilde{x} \tilde{x} \tilde{x}}=0 \text {, } \\
& \tilde{g}_{\tilde{x} \tilde{x} \tilde{y}}=0 \text {, } \\
& \widetilde{g}_{\tilde{x} \tilde{y} \tilde{y}}=0 \text {, } \\
& \tilde{\mathfrak{g}}_{\tilde{y} \tilde{y} \tilde{y}}=0 .
\end{aligned}
$$

According to Hopf bifurcation, the discriminatory quantity $s$ is given by

$$
\begin{aligned}
s= & -\operatorname{Re}\left[\frac{(1-2 \bar{\lambda}) \bar{\lambda}^{2}}{1-\lambda} \varphi_{11} \varphi_{20}\right]-\frac{1}{2}\left\|\varphi_{11}\right\|^{2}-\left\|\varphi_{02}\right\|^{2} \\
& +\operatorname{Re}\left(\bar{\lambda} \varphi_{21}\right),
\end{aligned}
$$



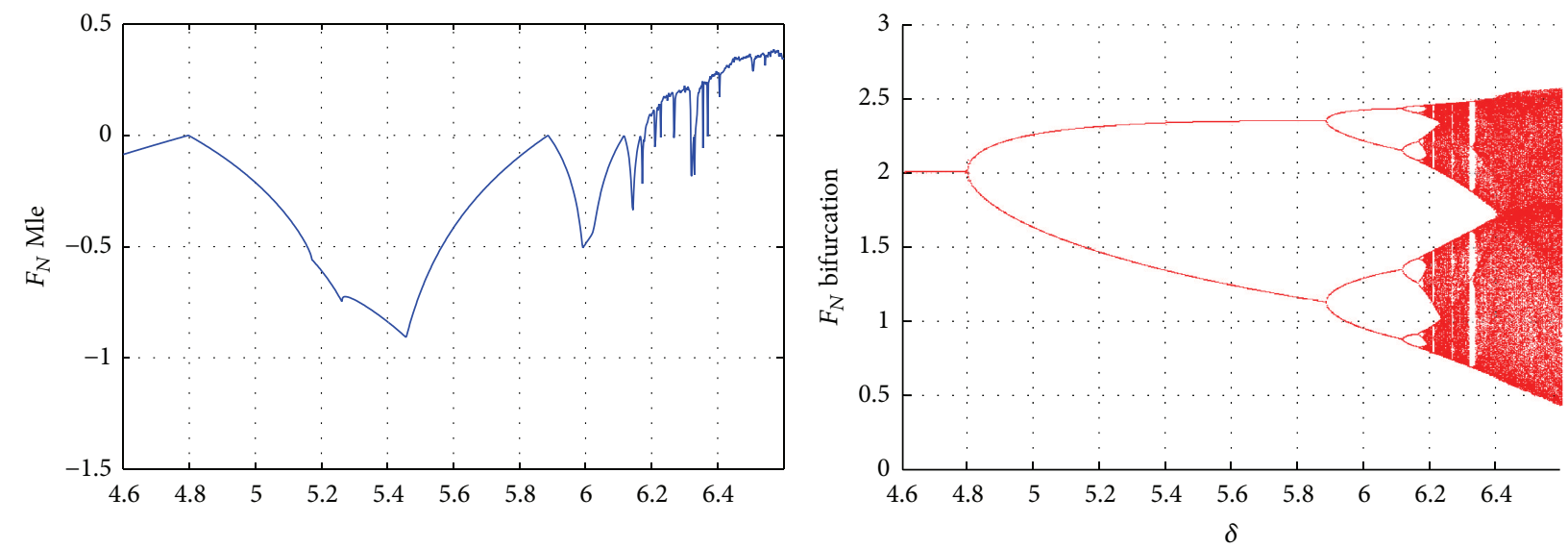

FiguRE 1: Largest Lyapunov exponents and bifurcation diagram of system (25) for $a=0.8, b=0.8, c=0.4, d=0.2, k=5, l=2$, and $A_{1}=0.18$ with the initial value of $(x, y)=(1,1)$ and $\delta$ covering $[4.6,6.6]$.
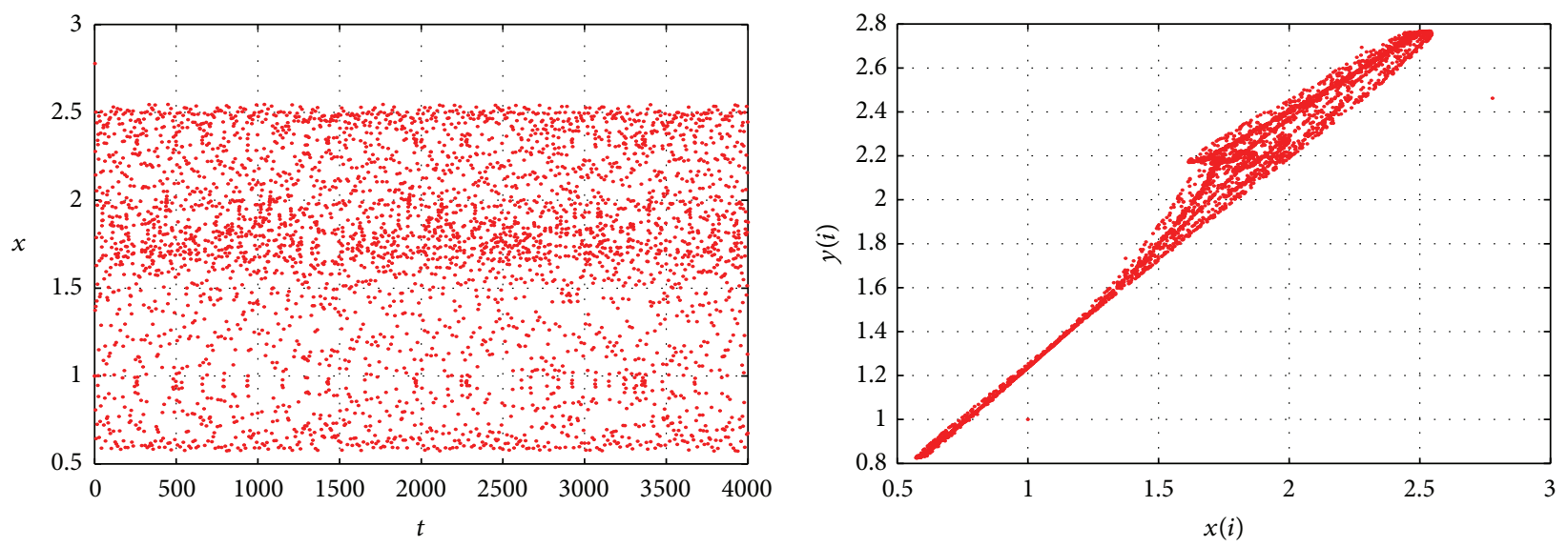

FIgURE 2: The portraits for several values of $A_{1}=0.18, \delta=6.45$.

where

$$
\begin{aligned}
& \varphi_{20}=\frac{1}{8}\left[\left(\widetilde{f}_{\tilde{x} \tilde{x}}-\tilde{f}_{\tilde{y} \tilde{y}}+2 \widetilde{g}_{\tilde{x} \tilde{y}}\right)\right. \\
& \left.+i\left(\widetilde{g}_{\tilde{x} \tilde{x}}-\tilde{g}_{\tilde{y} \tilde{y}}-2 \tilde{f}_{\tilde{x} \tilde{y}}\right)\right], \\
& \varphi_{11}=\frac{1}{4}\left[\left(\tilde{f}_{\tilde{x} \tilde{x}}+\widetilde{f}_{\tilde{y} \tilde{y}}\right)+i\left(\widetilde{g}_{\tilde{x} \tilde{x}}+\widetilde{g}_{\tilde{y} \tilde{y}}\right)\right], \\
& \varphi_{02}=\frac{1}{8}\left[\left(\widetilde{f}_{\tilde{x} \tilde{x}}-\widetilde{f}_{\tilde{y} \tilde{y}}-2 \widetilde{g}_{\tilde{x} \tilde{y}}\right)\right. \\
& \left.+i\left(\widetilde{g}_{\tilde{x} \tilde{x}}-\tilde{g}_{\tilde{y} \tilde{y}}+2 \tilde{f}_{\tilde{x} \tilde{y}}\right)\right], \\
& \varphi_{21}=\frac{1}{16}\left[\left(\tilde{f}_{\tilde{x} \tilde{x} \tilde{x}}+\tilde{f}_{\tilde{x} \tilde{y} \tilde{y}}+\widetilde{g}_{\tilde{x} \tilde{x} \tilde{y}}+\tilde{g}_{\tilde{y} \tilde{y} \tilde{y}}\right)\right. \\
& \left.+i\left(\widetilde{g}_{\tilde{x} \tilde{x} \tilde{x}}-\tilde{g}_{\tilde{x} \tilde{y} \tilde{y}}-\tilde{f}_{\tilde{x} \tilde{x} \tilde{y}}-\tilde{f}_{\tilde{y} \tilde{y} \tilde{y}}\right)\right] .
\end{aligned}
$$

Analyzing above and Hopf bifurcation conditions discussed in [30], we write the theorem as below.

Theorem 7. If the condition (38) holds, $s \neq 0$, and the parameter $\delta$ alters in the limited region of the point $(0,0)$, then system (38) passes through a Hopf bifurcation at the point $p_{3}\left(x^{*}, y^{*}\right)$. Moreover, if $s<0$ (resp., $\left.s<0\right)$, then an attracting (resp., repelling) invariant closed curve bifurcates from the fixed point $p_{3}\left(x^{*}, y^{*}\right)$ for $\delta>0$ (resp., $\left.\delta<0\right)$.

\section{Numerical Simulations}

To verify the theoretical analysis, we draw the bifurcation diagrams, largest Lyapunov exponents, and phase portraits for system (22). These diagrams show the complete dynamical behavior and the global stability of the system at the fixed points. The bifurcation behavior of system (22) is discussed in the following cases.

Case 1. On the one hand, if we take $a=0.8, b=0.8, c=$ $0.4, d=0.2, k=5, l=2, m=0.1$, and $A_{1}=0.18$ with the initial value of $(x, y)=(1,1)$ and $\delta$ covering $[4.6,6.6]$, then, we observe that at the fixed point $(2.0126,2.2006)$ a flip bifurcation appears at $\delta=4.792$. In this case $\gamma_{1}=-0.3528$, $\gamma_{2}=2.857$ (see Figure 1).

From Figure 2, we can see that the fixed point of system (9) is stable for $\delta<4.792$ and loses its stability at $\delta=4.792$ and there appears period doubling bifurcation for $\delta>4.792$. 

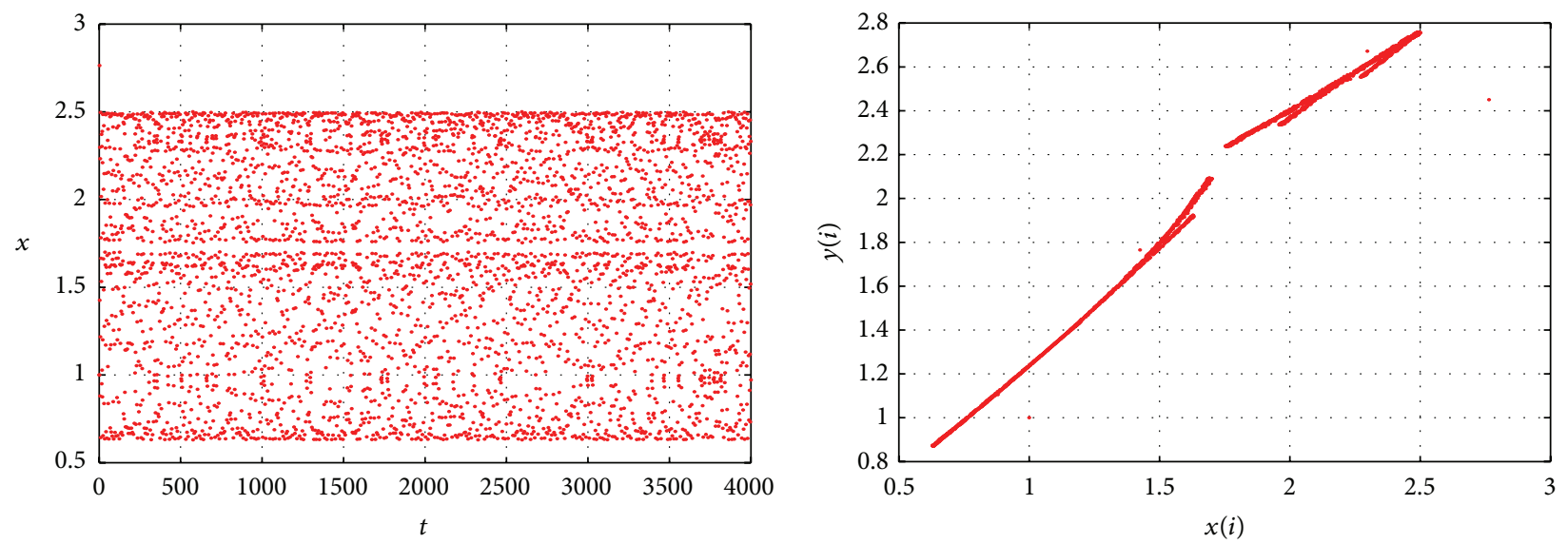

FIGURE 3: The portraits for several values of $A_{1}=0.18, \delta=6.4$.
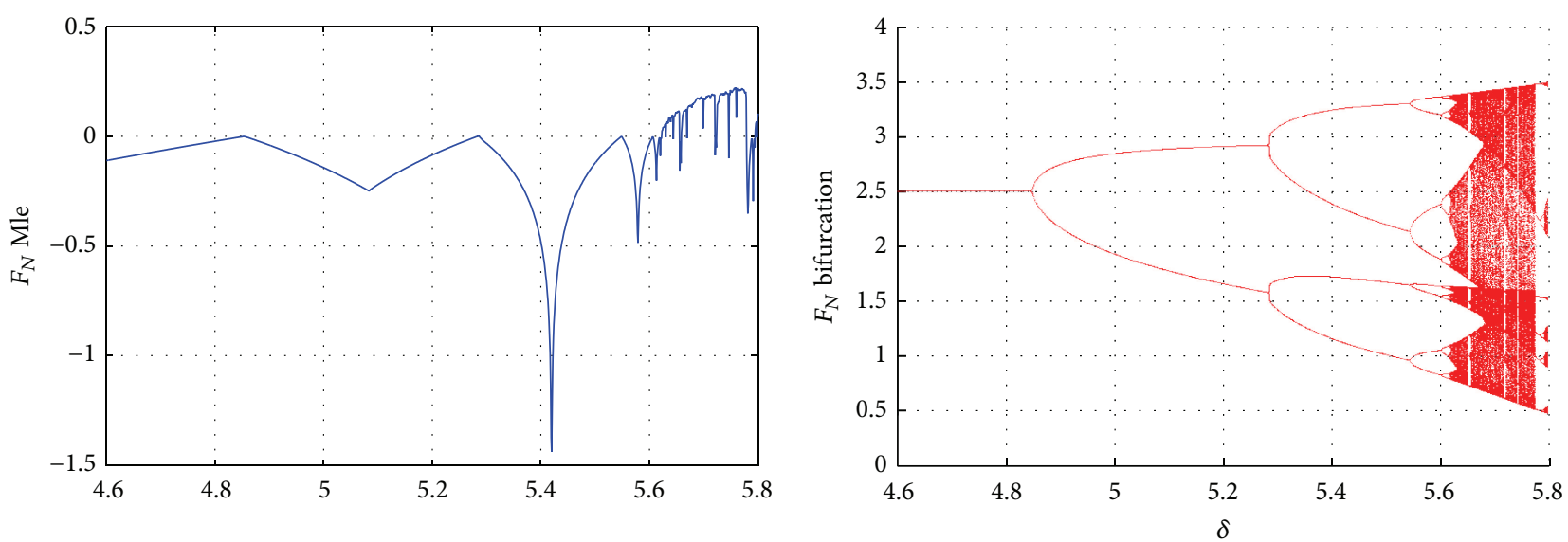

Figure 4: Largest Lyapunov exponents and bifurcation diagram of system (25) for $a=0.8, b=0.8, c=0.4, d=0.2, k=5, l=2$, and $A_{1}=0.03$ with the initial value of $(x, y)=(1,1)$ and $\delta$ covering $[4.6,5.8]$.

The phase portraits show that there are orbits of periods 2,4 , 8 for $\delta \in[5,6.15]$ and chaotic sets at $\delta=6.45$ and $\delta=6.4$ (see Figures 2 and 3 ).

On the other hand, if we take $a=0.8, b=0.8, c=0.4$, $d=0.2, k=5, l=2, m=0.1$, and $A_{1}=0.03$ with the initial value of $(x, y)=(1,1)$ and $\delta$ covering $[4.6,5.8]$, then, we observe that at the fixed point $(2.5025,2.2223)$ a flip bifurcation appears at $\delta=4.846$. In this case $\gamma_{1}=-0.175$, $\gamma_{2}=2.2893$ (see Figure 4).

From Figure 5, we can see that the fixed point of system (9) is stable for $\delta<4.846$ and loses its stability at $\delta=4.846$ and there appears period doubling bifurcation for $\delta>4.846$. The phase portraits show that there are orbits of periods 2,4 , 8 for $\delta \in[5,5.6]$ and chaotic sets at $\delta=5.7$ and $\delta=5.75$.

But, from the former paper in [17], we know when $\delta=$ 4.87985 loses its stability without Allee effect. So, we can see Allee effect changes the value of $\delta$.

Case 2. First, we take $a=0.8, b=0.8, c=0.4, d=0.2, k=5$, $l=3, m=0.1$, and $A_{1}=0.18$ with initial value of $(x, y)=$ $(1,1)$ and $\delta$ covering $[5.3,5.37]$ (see Figure 6$)$. We can see that at the fixed point $(3.0927,2.203)$ a Hopf bifurcation emerges at $\delta=5.335$. We can see that the fixed point of system (9) is stable $\delta<5.335$ and loses its stability at $\delta=5.335$, and an invariant circle appears at $\delta>5.335$ (see Figures 7 and 8). Here $\alpha=-0.8171, \beta=0.37345$, and $s=-0.911$.

Second, we take $a=0.8, b=0.8, c=0.4, d=0.2$, $k=5, l=3, m=0.1$, and $A_{1}=0.03$ with initial value of $(x, y)=(1,1)$ and $\delta$ covering $[4.87,4.95]$ (see Figure 9). We can see that at the fixed point $(3.206,2.207)$ a Hopf bifurcation emerges at $\delta=4.8795$. We can see that the fixed point of system (9) is stable $\delta<4.8795$ and loses its stability at $\delta=4.8795$, and an invariant circle appears at $\delta>4.8795$ (see Figures 10 and 11). Here $\alpha=-0.9523, \beta=0.3096$, and $s=-0.9172$.

\section{Conclusions}

In recent years, the Allee effect has drawn considerable attention in almost every aspect of ecology and conservation. The consequences of Allee effect become more and more significant. In this paper, based on the discrete-time predator-prey system with predator partially dependent on prey and crowding effect of predator, we investigated the impact of Allee 

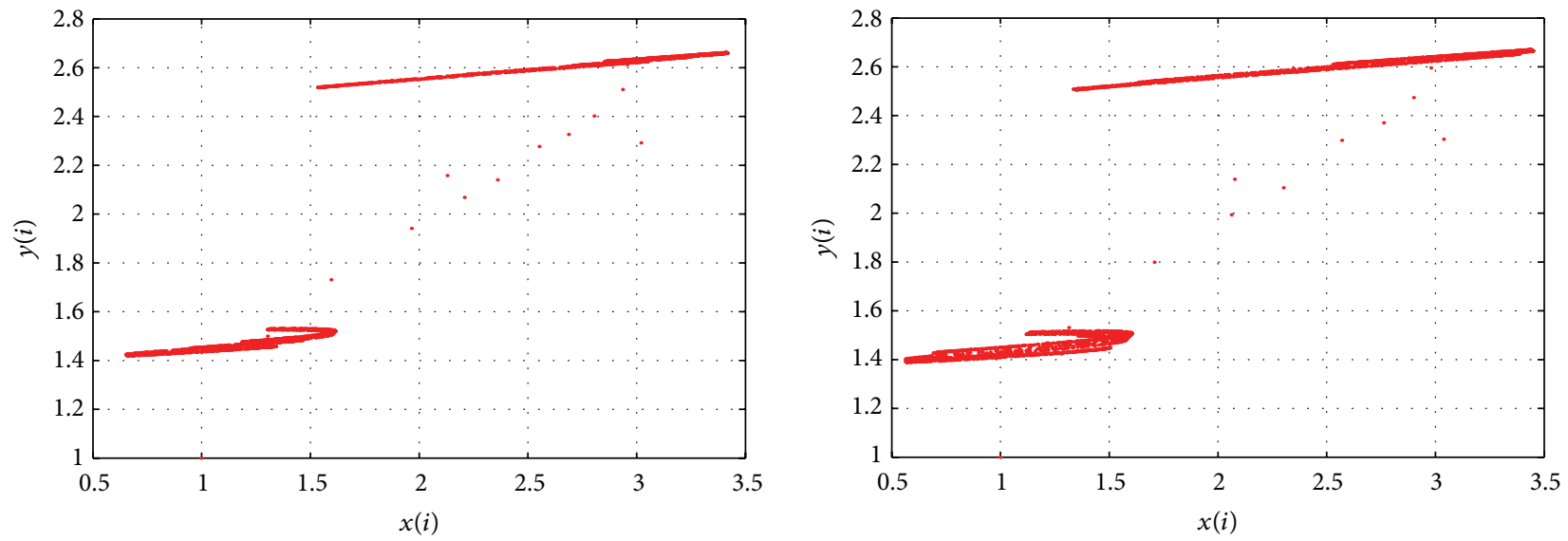

FIGURE 5: The portraits for several values of $A_{1}=0.03, \delta=5.7$, and $\delta=5.75$.
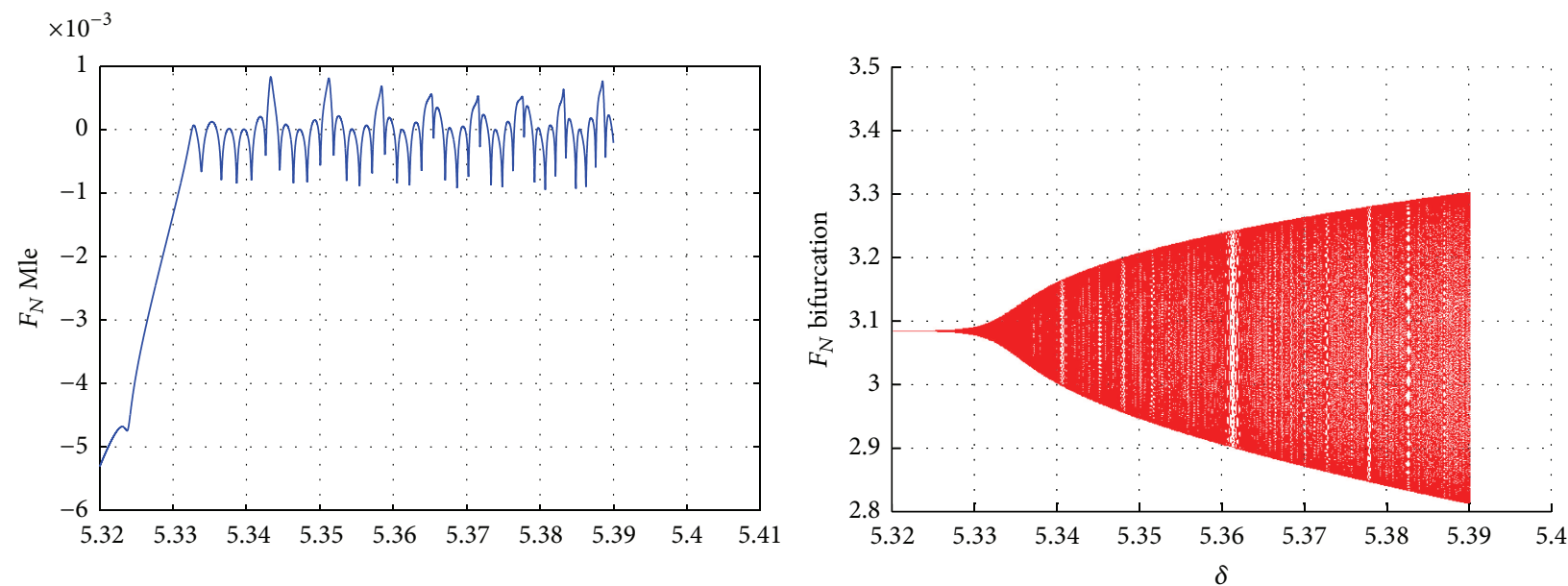

Figure 6: Largest Lyapunov exponents and bifurcation diagram of system (25) for $a=0.8, b=0.8, c=0.4, d=0.2, k=5, l=3$, and $A_{1}=0.18$ with the initial value of $(x, y)=(1,1)$ and $\delta$ covering $[5.32,5.39]$.
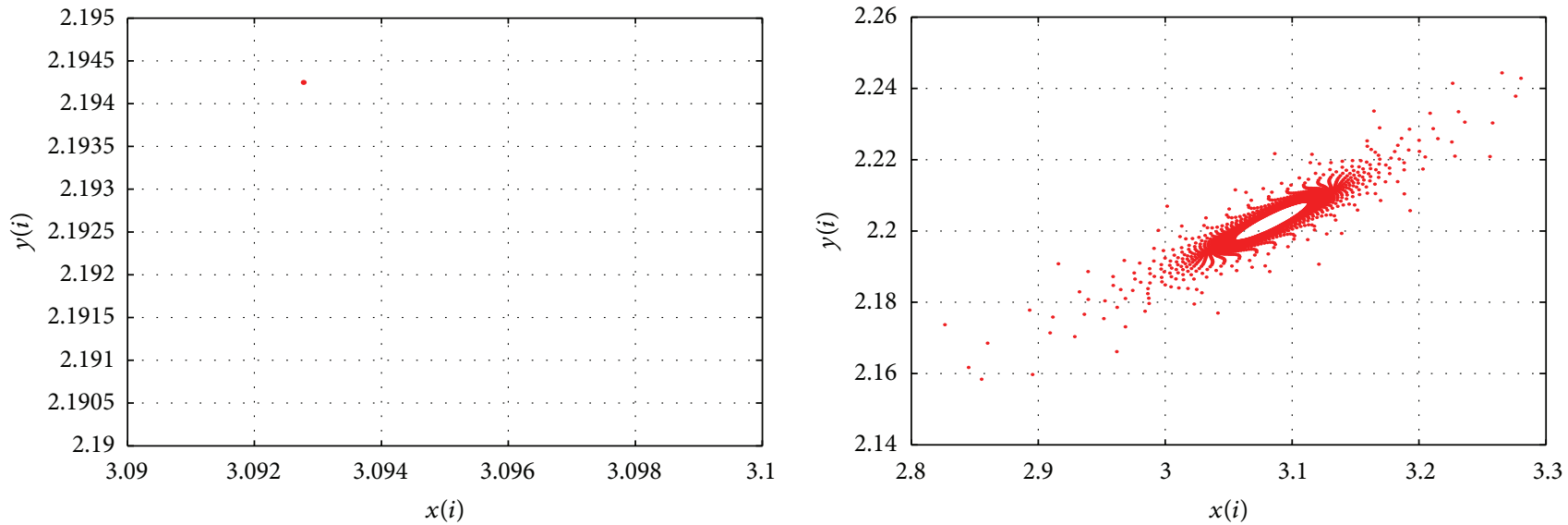

FIgURE 7: The portraits for several values of $A_{1}=0.18, \delta=5.3$, and $\delta=5.335$. 

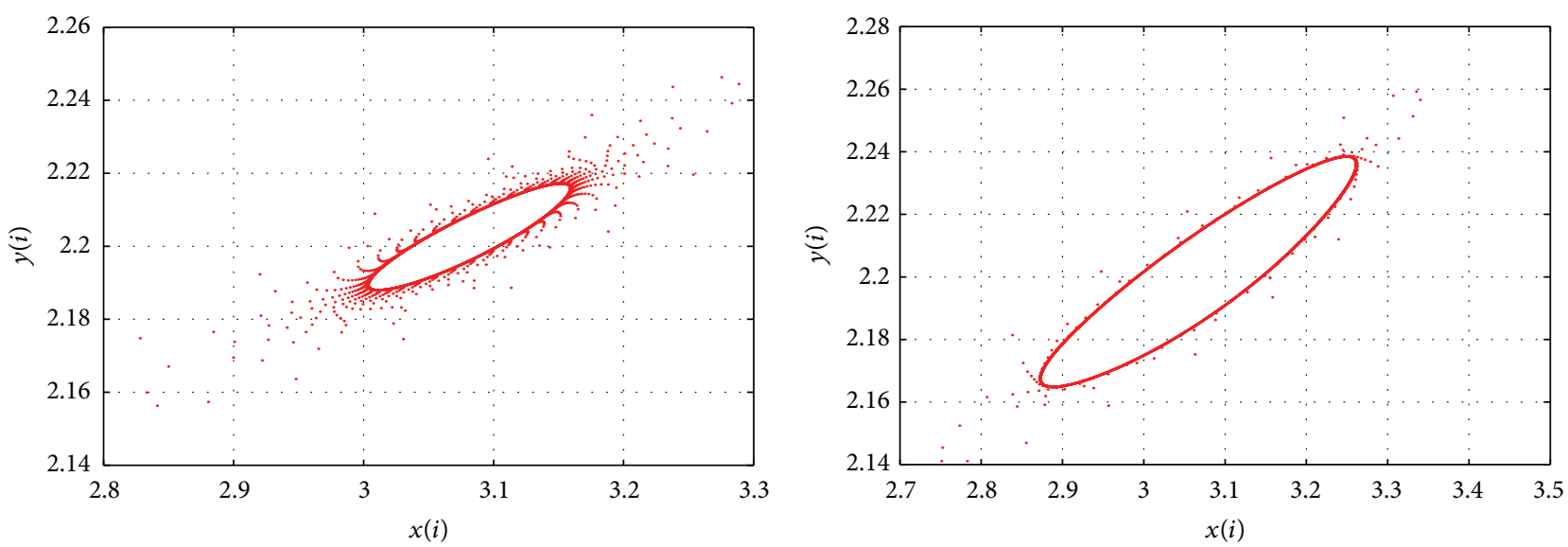

FIgURE 8: The portraits for several values of $A_{1}=0.18, \delta=5.34$, and $\delta=5.37$.
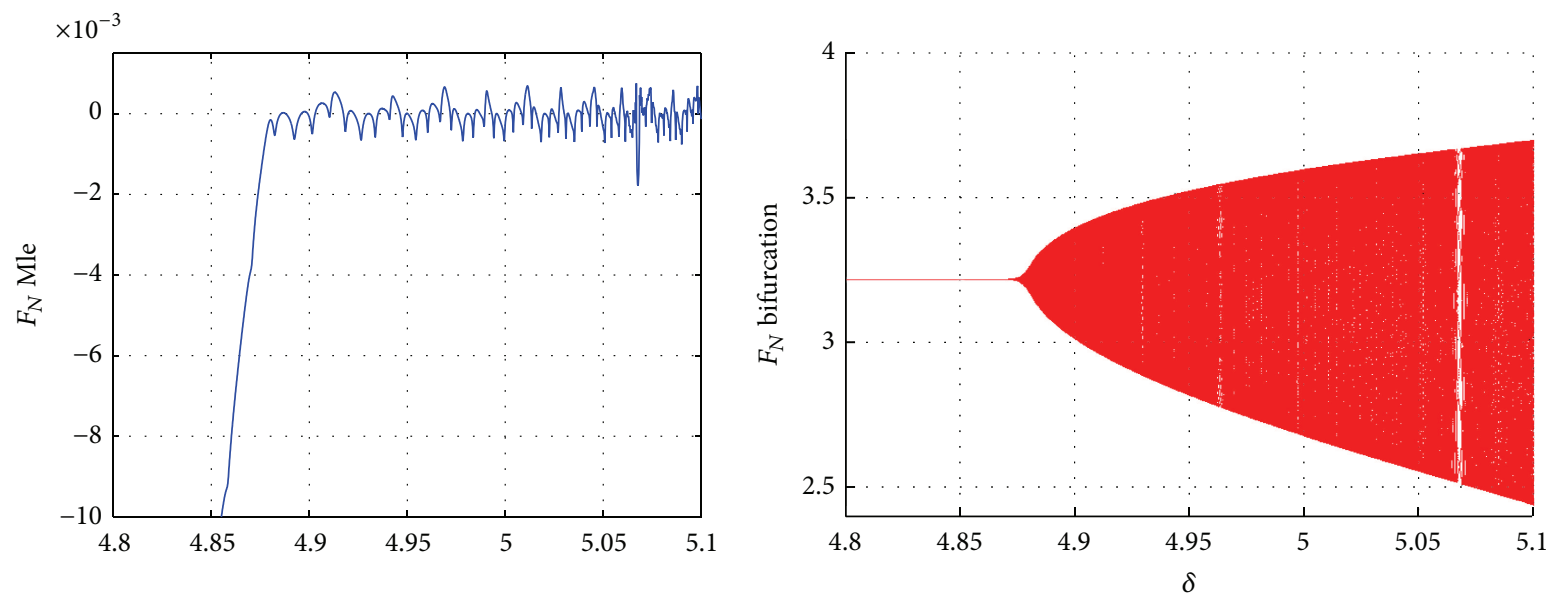

FIGURE 9: Largest Lyapunov exponents and bifurcation diagram of system (25) for $a=0.8, b=0.8, c=0.4, d=0.2, k=5, l=3$, and $A_{1}=0.03$ with the initial value of $(x, y)=(1,1)$ and $\delta$ covering $[4.8,5.1]$.
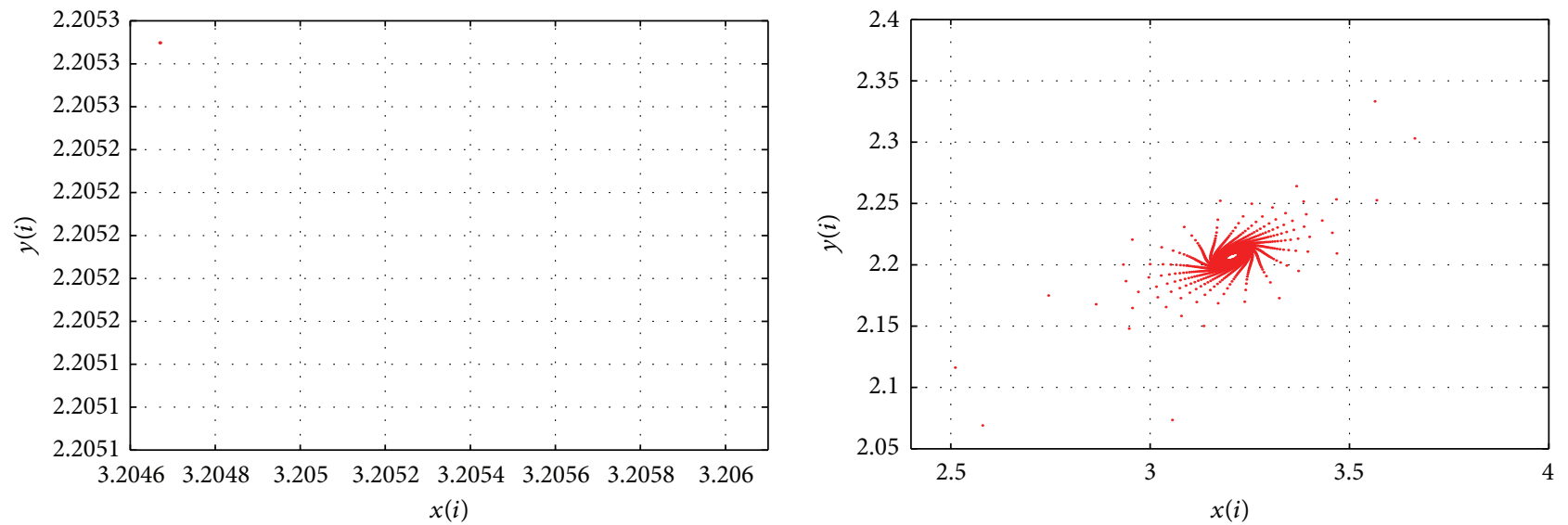

Figure 10: The portraits for several values of $A_{1}=0.03, \delta=4.875$, and $\delta=4.8795$. 

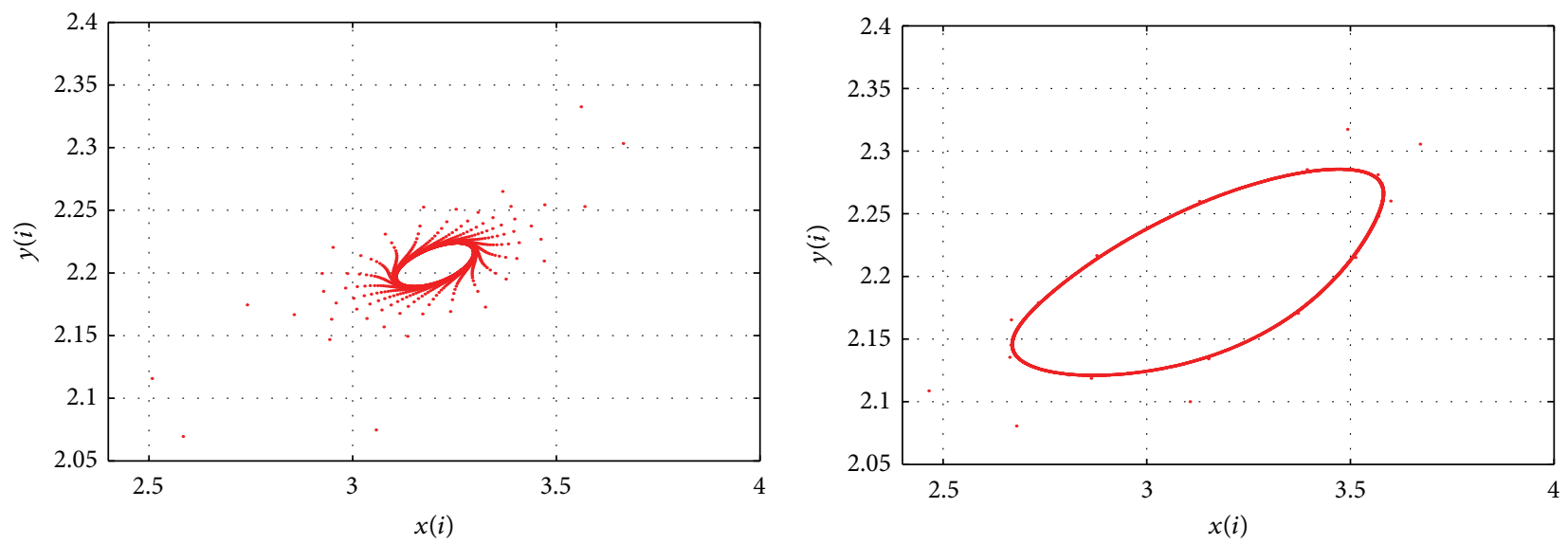

FIGURE 11: The portraits for several values of $A_{1}=0.03, \delta=4.885$, and $\delta=5$.

effect on the stability of a predator-prey system when the species is subject to Allee effect in the closed first quadrant $R_{+}^{2}$.

By analyzing system (9), we found that the system is asymptotically stable at the $p_{2}(0, c / d), p_{3}\left(x^{*}, y^{*}\right)$ at the interior of the first quadrant for any parameter values. Ecologically speaking, this means that there only exists predator at $p_{2}(0, c / d)$, in which $\delta$ satisfies $0<\delta<\min \{2 / c, 2 d l / b c\}$, and the prey and predator species coexist at $p_{3}\left(x^{*}, y^{*}\right)$, which should satisfy either condition (1): $G^{2}-4 H \geq 0$ and $0<$ $\delta<\left(-G-\sqrt{G^{2}-4 H}\right) / H$ or condition (2): $G^{2}-4 H<0$ and $0<\delta<-G / H$.

It is shown that system (9) with Allee effect can go through flip bifurcation and Hopf bifurcation at the neighborhood of the equilibrium point. Because one of the eigen values of fixed point $p_{1}(k, 0)$ is -1 and magnitude of other is not equal to 1 when $\delta=2 / a+2 A_{1} / a k$, a flip bifurcation occurs when a parameter changes in small neighborhood of $\delta=2 / a+2 A_{1} / a k$. The point $p_{2}(0, c / d)$ undergoes flip bifurcation when the parameter changes in small neighborhood of $F_{A}=\left\{\left(a, b, c, d, k, l, m, \delta, A_{1}\right), \delta=\right.$ $\left.2 / c, \delta \neq 2 d l / b c, a, b, c, d, k, l, m, \delta, A_{1}>0\right\}$. Finally, at the positive equilibrium point $p_{3}\left(x^{*}, y^{*}\right)$, by our analysing a flip bifurcation will occur in the neighborhood of $F_{B 1}=$ $\left\{\left(a, b, c, d, k, l, m, \delta, A_{1}\right): \delta=\left(-G-\sqrt{G^{2}-4 H}\right) / H\right.$, $G^{2}-4 H \geq 0$ and $\left.a, b, c, d, k, l, m, \delta, A_{1}>0\right\}$ or $F_{B 2}=$ $\left\{\left(a, b, c, d, k, l, m, \delta, A_{1}\right): \delta=\left(-G+\sqrt{G^{2}-4 H}\right) / H, G^{2}-4 H \geq\right.$ 0 and $\left.a, b, c, d, k, l, m, \delta, A_{1}>0\right\}$. In contrast, the system will get through a Hopf bifurcation at the neighborhood of $H_{B}=\left\{\left(a, b, c, d, k, l, m, \delta, A_{1}\right): \delta=-G / H, G^{2}-4 H<0\right.$ and $\left.a, b, c, d, k, l, m, \delta, A_{1}>0\right\}$.

In the paper, we assume the parameter $A_{1}>0$; that is, we only consider the strong Allee effect. When $A_{1}<0$, it is the weak Allee effect, and it is not the same as the $A_{1}>0$. This is the situation we have to consider in the future.

\section{Competing Interests}

The authors declare that there is no conflict of interests regarding the publication of this paper.

\section{Acknowledgments}

This work was supported by the National Natural Science Foundation of Henan Education committee (Grant no. 201113110034) and Science and Technology Program of Henan Province (Grant no. 162102310604).

\section{References}

[1] A. A. Berryman, "The origins and evolution of predator-prey theory," Ecology, vol. 73, no. 5, pp. 1530-1535, 1992.

[2] J. Dhar, "A prey-predator model with diffusion and a supplementary resource for the prey in a two-patch environment," Mathematical Modelling and Analysis, vol. 9, no. 1, pp. 9-24, 2004.

[3] J. Dhar and K. S. Jatav, "Mathematical analysis of a delayed stage-structured predator-prey model with impulsive diffusion between two predators territories," Ecological Complexity, vol. 16, pp. 59-67, 2013.

[4] B. Dubey, "A prey-predator model with a reserved area," Nonlinear Analysis: Modelling and Control, vol. 12, no. 4, pp. 479-494, 2007.

[5] H. Freedman, Deterministic Mathematical Models in Population Ecology, HIFR Consulting, Edmonton, Canada, 1980.

[6] J. M. Jeschke, M. Kopp, and R. Tollrian, "Predator functional responses: discriminating between handling and digesting prey," Ecological Monographs, vol. 72, no. 1, pp. 95-112, 2002.

[7] R. E. Kooij and A. Zegeling, "A predator-prey model with Ivlev's functional response," Journal of Mathematical Analysis and Applications, vol. 198, no. 2, pp. 473-489, 1996.

[8] A. J. Lotka, Scientific Books: Elements of Physical Biology, vol. 66, pp. 281-282, 1927.

[9] W. Ma and Y. Takeuchi, "Stability analysis on a predator-prey system with distributed delays," Journal of Computational and Applied Mathematics, vol. 88, no. 1, pp. 79-94, 1998.

[10] R. M. May, Stability and Complexity in Model Ecosystems, vol. 6, Princeton University Press, 2001.

[11] M. Sen, M. Banerjee, and A. Morozov, "Bifurcation analysis of a ratio-dependent prey-predator model with the Allee effect," Ecological Complexity, vol. 11, pp. 12-27, 2012. 
[12] S. Sinha, O. P. Misra, and J. Dhar, "Modelling a predator-prey system with infected prey in polluted environment," Applied Mathematical Modelling, vol. 34, no. 7, pp. 1861-1872, 2010.

[13] V. Volterra, "Fluctuations in the abundance of a species considered mathematically," Nature, vol. 118, no. 2972, pp. 558-560, 1926.

[14] J. Dhar, H. Singh, and H. S. Bhatti, "Discrete-time dynamics of a system with crowding effect and predator partially dependent on prey," Applied Mathematics and Computation, vol. 252, pp. 324-335, 2015.

[15] W. Allee, Animal Aggregations: A Study in General Sociology, University of Chicago Press, Chicago, Ill, USA, 1931.

[16] E. D. Conway and J. A. Smoller, "Global analysis of a system of predator-prey equations," SIAM Journal on Applied Mathematics, vol. 46, no. 4, pp. 630-642, 1986.

[17] A. D. Bazykin, Nonlinear Dynamics of Interacting Populations, vol. 11 of World Scientific Series on Nonlinear Science Series A, World Scientific, River Edge, NJ, USA, 1998.

[18] E. González-Olivares, J. Mena-Lorca, A. Rojas-Palma, and J. D. Flores, "Dynamical complexities in the Leslie-Gower predatorprey model as consequences of the Allee effect on prey," Applied Mathematical Modelling, vol. 35, no. 1, pp. 366-381, 2011.

[19] P. J. Pal and P. K. Mandal, "Bifurcation analysis of a modified Leslie-Gower predator-prey model with Beddington-DeAngelis functional response and strong Allee effect," Mathematics and Computers in Simulation, vol. 97, pp. 123-146, 2014.

[20] L. Berec, E. Angulo, and F. Courchamp, "Multiple Allee effects and population management," Trends in Ecology and Evolution, vol. 22, no. 4, pp. 185-191, 2007.

[21] D. S. Boukal and L. Berec, "Single-species models of the Allee effect: extinction boundaries, sex ratios and mate encounters," Journal of Theoretical Biology, vol. 218, no. 3, pp. 375-394, 2002.

[22] D. S. Boukal, M. W. Sabelis, and L. Berec, "How predator functional responses and Allee effects in prey affect the paradox of enrichment and population collapses," Theoretical Population Biology, vol. 72, no. 1, pp. 136-147, 2007.

[23] G. Wang, X.-G. Liang, and F.-Z. Wang, "The competitive dynamics of populations subject to an Allee effect," Ecological Modelling, vol. 124, no. 2-3, pp. 183-192, 1999.

[24] E. González-Olivares, B. González-Yañez, J. Mena-Lorca, and R. Ramos-Jiliberto, "Modelling the Allee effect: are the different mathematical forms proposed equivalents?" in Proceedings of the International Symposium on Mathematical and Computational Biology, R. Mondaini, Ed., pp. 53-71, E-Papers Serviços Editoriais Ltda, Rio de Janeiro, Brazil, 2007.

[25] F. Courchamp, T. Clutton-Brock, and B. Grenfell, "Inverse density dependence and the Allee effect," Trends in Ecology and Evolution, vol. 14, no. 10, pp. 405-410, 1999.

[26] P. A. Stephens and W. J. Sutherland, "Consequences of the Allee effect for behaviour, ecology and conservation," Trends in Ecology and Evolution, vol. 14, no. 10, pp. 401-405, 1999.

[27] J. Zu, "Global qualitative analysis of a predator-prey system with Allee effect on the prey species," Mathematics and Computers in Simulation, vol. 94, pp. 33-54, 2013.

[28] X. Liu and D. Xiao, "Complex dynamic behaviors of a discretetime predator-prey system," Chaos, Solitons \& Fractals, vol. 32, no. 1, pp. 80-94, 2007.

[29] Z. He and X. Lai, "Bifurcation and chaotic behavior of a discretetime predator-prey system," Nonlinear Analysis: Real World Applications, vol. 12, no. 1, pp. 403-417, 2011.
[30] J. Guckenheimer and P. Holmes, Nonlinear Oscillations, Dynamical Systems, and Bifurcations of Vector Fields, vol. 42 of Applied Mathematical Sciences, Springer, New York, NY, USA, 1983. 


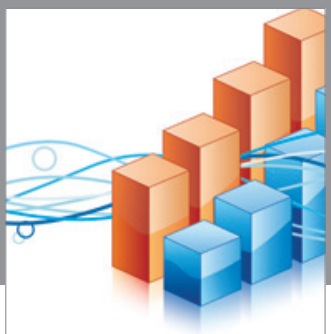

Advances in

Operations Research

vatem alat4

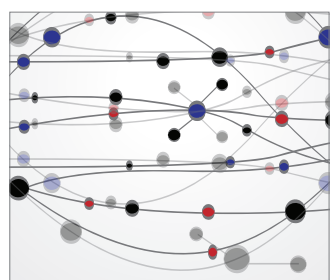

\section{The Scientific} World Journal
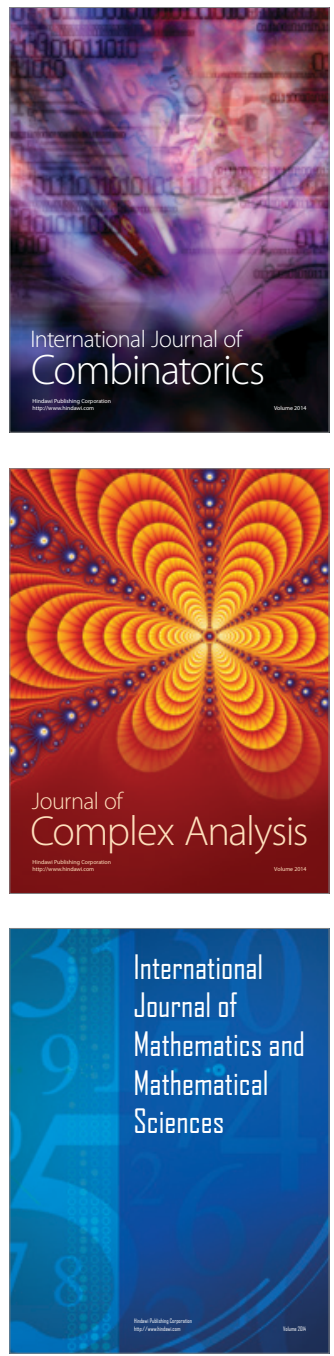
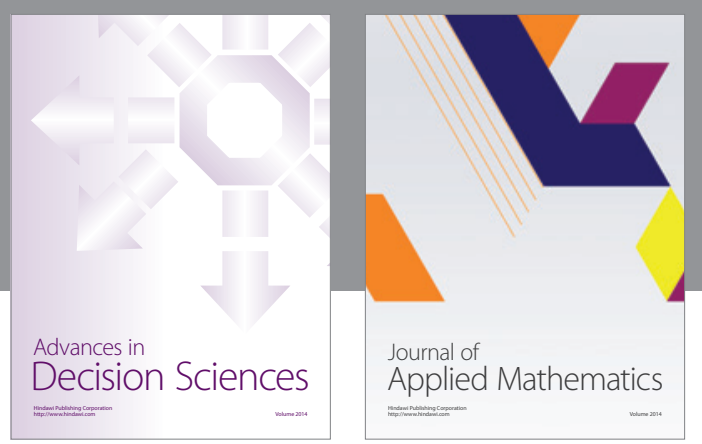

Algebra

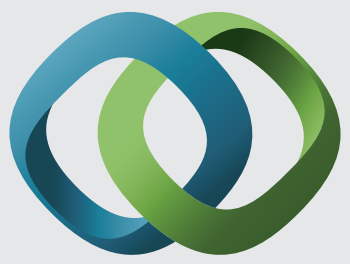

\section{Hindawi}

Submit your manuscripts at

http://www.hindawi.com
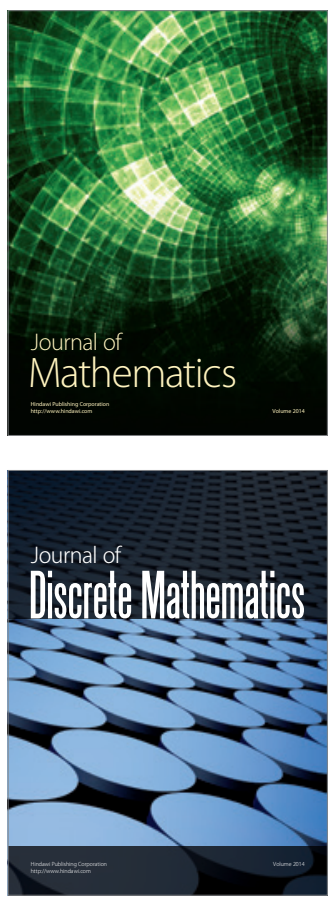

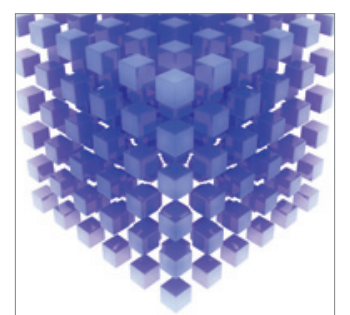

Mathematical Problems in Engineering
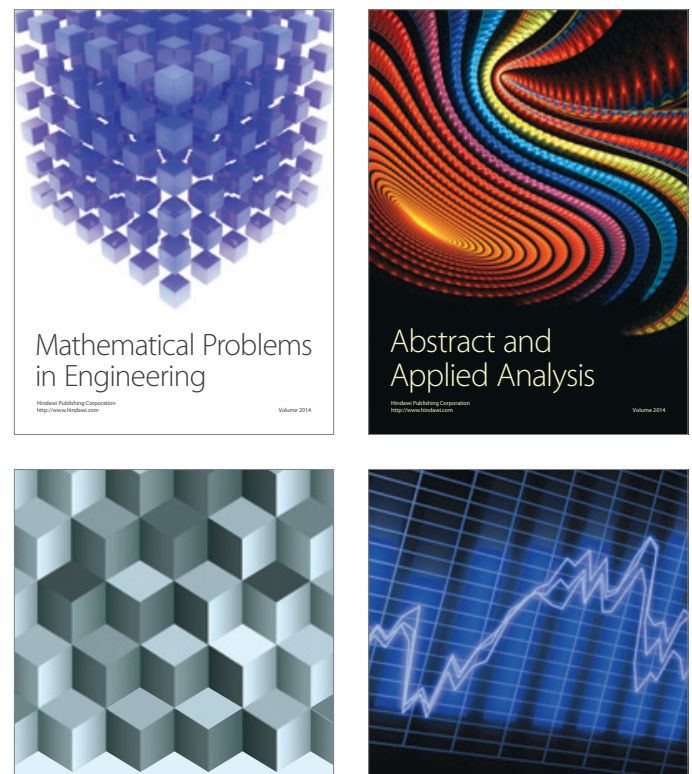

Journal of

Function Spaces

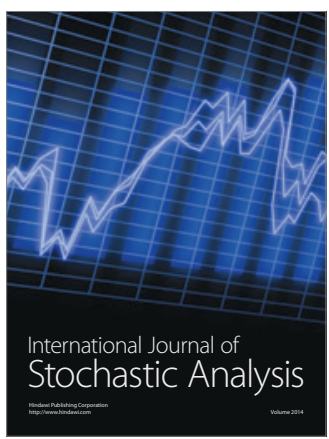

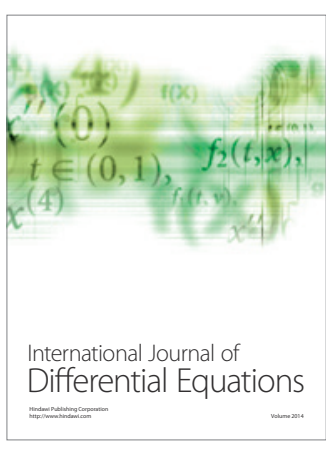
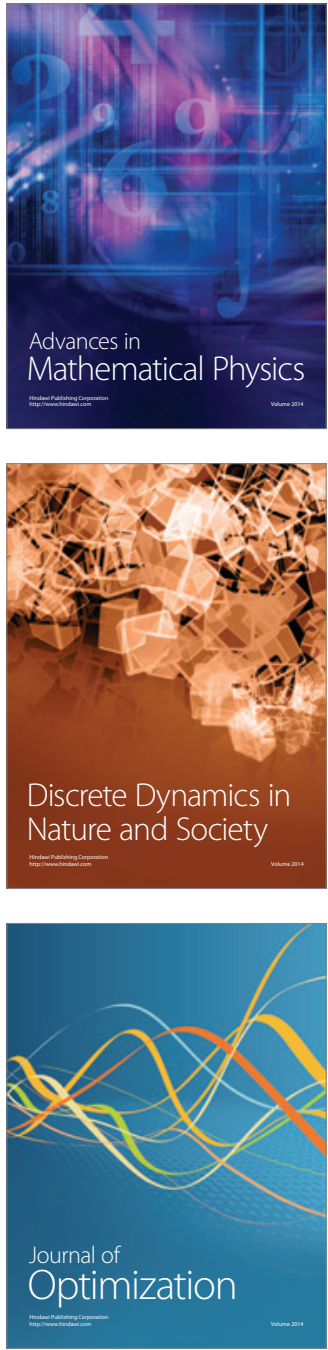